\title{
$\alpha_{2}$-Macroglobulin and eosinophil cationic protein in the allergic airway mucosa in seasonal allergic rhinitis
}

\author{
P. Meyer*, C.G.A. Persson**, M. Andersson***, P. Wollmer ${ }^{+}$, M. Linden*, C. Svensson***, L. Greiff***
}

$\alpha_{2}$-Macroglobulin and eosinophil cationic protein in the allergic airway mucosa in seasonal allergic rhinitis. P. Meyer, C.G.A. Persson, M. Andersson, P. Wollmer, M. Linden, C. Svensson, L. Greiff. (C) ERS Journals Ltd 1999.

ABSTRACT: As previously demonstrated in seasonal allergic rhinitis, increased microvascular permeability and eosinophil activation are key features of allergic airway inflammation. In the present study, the hypothesis that exudation of $\alpha_{2}$-macroglobulin may cause the appearance of eosinophil cationic protein (ECP) in the airway lumen was explored.

Nasal lavages were carried out using the nasal pool device before and during the pollen season both at baseline and after histamine challenge in 10 children with allergic rhinitis. Nasal lavage fluid levels of $\alpha_{2}$-macroglobulin and ECP were determined.

All patients experienced nasal symptoms of allergic rhinitis during the pollen season $(p<0.01-0.05)$. Baseline nasal lavage fluid levels of $\alpha_{2}$-macroglobulin and ECP were increased during the season $(p<0.01-0.05)$ and were found to be well correlated $(\mathbf{p}<\mathbf{0 . 0 0 0 1})$. Histamine produced concentration-dependent plasma exudation before and during the pollen season, but it was only during the pollen season that this caused an increase in the lavage fluid levels of ECP $(p<0.05)$.

These data suggest that exudation of plasma and increased tissue levels and output of eosinophil cationic protein characterize nasal mucosal inflammation in children with seasonal allergic rhinitis. The plasma exudation process in part may account for lumenal entry of eosinophil cationic protein molecules that have been released in mucosal tissue compartments. A combination of induced exudation and nasal lavage may improve the yield of important markers of inflammation in studies of nasal diseases.

Eur Respir J 1999; 13: 633-637.
Depts of *Paediatrics, **Clinical Pharmacology, ***Otorhinolaryngology and ${ }^{+}$Clinical Physiology, University Hospital, Lund/Malmö, Sweden. "Dept of Research and Development, Astra Draco, Lund, Sweden.

Correspondence: L. Greiff

Dept of Otorhinolaryngology

Head and Neck Surgery

University Hospital

S-221 85 Lund

Sweden

Fax: 46462110968

\section{Keywords: Airway}

inflammation

paediatric

plasma exudation

rhinitis

Received: April 281998

Accepted after revision October 271998

Supported by the Swedish Medical Research Council (project 8308), the Vardal Foundation, the Swedish Association against Asthma and Allergy, and the Medical Faculty of Lund University.
Microvascular permeability and eosinophil activation, as indicated by plasma proteins (e.g. albumin, fibrinogen and $\alpha_{2}$-macroglobulin), and eosinophil cationic protein (ECP) in nasal discharges may characterize allergic rhinitis and reflect disease severity. In accord, nasal lavage fluid levels of these indices are increased in seasonal allergic rhinitis in adults [1-3]. However, there is as yet limited information about the occurrence of plasma exudation and eosinophil activation in children suffering from this disease. The "nasal pool" device was recently introduced for challenge and lavage of the nasal airway mucosa in children [4]. Thus, it now appears feasible to explore several aspects of the exudative and eosinophilic inflammation in children with seasonal allergic rhinitis, including the use of nasal histamine challenges to produce acute effects such as lumenal entry of bulk plasma [4]. This study tests a novel hypothesis of luminal entry of ECP in human airways.

In preliminary experiments in adults it was observed that histamine challenge-induced plasma exudation responses may move other, non-plasma-derived, subepithelial molecules such as interleukin (IL)-6 into the airway lumen [5]. The acute lumenal appearance of cellular indices during exudation is in line with data demonstrating extensive lamina propria and epithelial passage of extravasated bulk plasma within $10 \mathrm{~min}$ after topical mucosal challenge with histamine-like mediators [6]. Plasma proteins such as $\alpha_{2}$ macroglobulin can bind ECP as well as many cytokines [7, 8], and hence, it was hypothesized [9] that induced exudation may cause important mucosal indices of allergic inflammation to appear in the nasal lavage fluid.

In the present study, involving 7-11 yr-old children with seasonal allergic rhinitis, nasal lavages were carried out to assess baseline changes during a pollen season and histamine challenge was combined with nasal lavage both before and during the season. Nasal lavage fluid levels of $\alpha_{2}$-macroglobulin and ECP have been determined as indices of mucosal exudation of plasma and eosinophil activation, respectively. In particular, the study explored the possibility of a parallel relation in the appearance of $\alpha_{2}$ macroglobulin and ECP in order to elucidate the possibility that an acute exudation response may increase the lavage fluid levels of ECP.

\section{Materials and methods}

\section{Study design}

Children with seasonal allergic rhinitis were examined during the 1996 Swedish pollen season. Nasal isotonic saline lavages were carried out before and on four occasions during the season. Furthermore, nasal histamine 
challenges were performed before and once during the season. The lavage fluid levels of $\alpha_{2}$-macroglobulin and ECP were measured as indices of plasma exudation and eosinophil activation, respectively. No drugs were allowed during the pollen season except for the rescue medication, loratadine tablets (Clarityn $\mathbb{R}$, Schering-Plough, Stock-

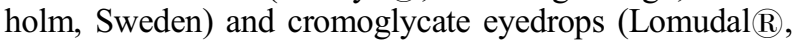
Rhone-Poulenc Rorer, Helsingborg, Sweden) in clinical doses. The patients were instructed to use the rescue medication if more than moderate symptoms occurred.

\section{Patients}

Ten children (four male and six female, aged 7-11 yrs, mean age 9 yrs) participated in the study. The children had a history of seasonal birch pollen allergic rhinitis, which was verified by a positive skin prick test (five patients also presented positive skin prick tests to timothy pollen allergen). The children had no history of perennial nasal or bronchial disease, recent respiratory tract infection or recent drug treatment. The study was approved by the local ethics committee and informed consent was obtained from the patients and their parents/guardians.

\section{Symptom scores}

Nasal symptoms, i.e. rhinorrhea, blockage and sneezes, as well as eye symptoms, were scored by the children once every day during the pollen season: score 0 , none; 1 , mild; 2, moderate; and 3, severe symptoms. Chest symptoms were not scored during the season. However, the patients and their parents were specifically questioned at the final visit as to whether chest symptoms had occurred during the study period.

\section{Nasal pool challenge and lavage technique}

The "nasal pool" device was used for saline lavages and for concomitant histamine challenge and lavage of the nasal mucosa [4]. The nasal pool device is a compressible plastic container equipped with a nasal adapter. The adapter is inserted into a nostril and the container is compressed by the sitting subject leaning forward in a $60^{\circ}$ flexed neck position. The nasal pool fluid is then instilled in one of the nasal cavities and maintained in contact with a large and reproducible area of the mucosal surface for a determined period (in this study, $2 \mathrm{~min}$ and $5 \mathrm{~min}$ ). When the pressure on the device is released the fluid returns into the container. In the present study, the volume of nasal pool fluid was $12 \mathrm{~mL}$.

\section{Isotonic saline lavage of the nasal mucosa}

Two-minute lavages with isotonic saline were performed before the pollen season and on four occasions during the season. These lavages were carried out on the same days for all patients and at the same time point, i.e. in the mornings. The recovered lavage fluids were frozen $\left(-20^{\circ} \mathrm{C}\right)$ for later analysis of $\alpha_{2}$-macroglobulin and ECP. Based on the relative inability of the children and their patients to visit the clinic on multiple occasions, three of the baseline lavages (lavage 2, 3 and 4) were carried out at home under the supervision of their parents and the lavage fluids were, therefore, frozen without prior centrifugation. For conformity, the 2-min lavages that preceded the histamine challenge series (lavages 1 and 5) (carried out at the clinic) were also processed without prior centrifugation.

\section{Histamine challenge and lavage}

Immediately after the baseline 2-min lavages performed before the pollen season and at the fourth occasions during the season, a 5-min lavage with isotonic saline was performed. With 5-min intervals, this lavage was then followed by first a histamine $\left(40 \mu \mathrm{g} \cdot \mathrm{mL}^{-1}\right)$ lavage and then a histamine $\left(400 \mu \mathrm{g} \cdot \mathrm{mL}^{-1}\right)$ lavage. The recovered lavage fluids (whole samples) were centrifuged $(105 \times \mathrm{g}, 10 \mathrm{~min}$, $4{ }^{\circ} \mathrm{C}$ ) and aliquots were prepared from the supernatants and frozen $\left(-20^{\circ} \mathrm{C}\right)$ for later analysis of $\alpha_{2}$-macroglobulin and ECP.

\section{Analysis of $\alpha_{2}$-macroglobulin and eosinophil cationic protein}

The lavage fluids were placed in coded vials and the levels of $\alpha_{2}$-macroglobulin were measured using a radioimmunoassay sensitive to $7.8 \mathrm{ng} \cdot \mathrm{mL}^{-1}$. Rabbit anti-human $\alpha_{2}$-macroglobulin (Dakopatts, Copenhagen, Denmark) was used as antiserum and human serum (Behringwerke Diagnostica, Marburg, Germany) as standard. Human $\alpha_{2}$-macroglobulin (Cappel-Organon Teknika, Turnhout, Belgium) was iodinated using the lactoperoxidase method. Tracer and standard (or sample) were mixed with anti-serum before adding goat anti-rabbit antiserum (Astra Draco, Lund, Sweden). The bound fraction was measured using a gamma counter. The intra-assay and interassay coefficients of variation were between $3.8-6.0 \%$ and $3.1-7.2 \%$, respectively.

The nasal lavage fluid levels of ECP were measured by a fluoroimmunoassay using the CAP system (Pharmacia, Uppsala, Sweden). Both the intra-assay and interassay coefficients of variation are, according to the supplier, $<10 \%$, and the cross-reactivity to eosinophil protein $\mathrm{X}$ $<0.02 \%$.

\section{Statistics}

Differences in baseline lavage fluid levels of $\alpha_{2}$-macroglobulin and ECP were examined using the Friedman test. If statistical significance emerged, further analyses were performed using the Wilcoxon signed rank test. Differences in saline and histamine-induced lavage fluid levels of $\alpha_{2}$-macroglobulin and ECP, respectively, between observations before and during the pollen season were examined using the Wilcoxon signed rank test. Correlations between $\alpha_{2}$-macroglobulin and ECP were examined using the Spearman rank correlation test. A p-value $<0.05$ was considered significant. Data are presented as mean \pm SEM.

\section{Results}

The birch pollen counts demonstrated a marked but short birch pollen season (fig. 1a). The children experienced 

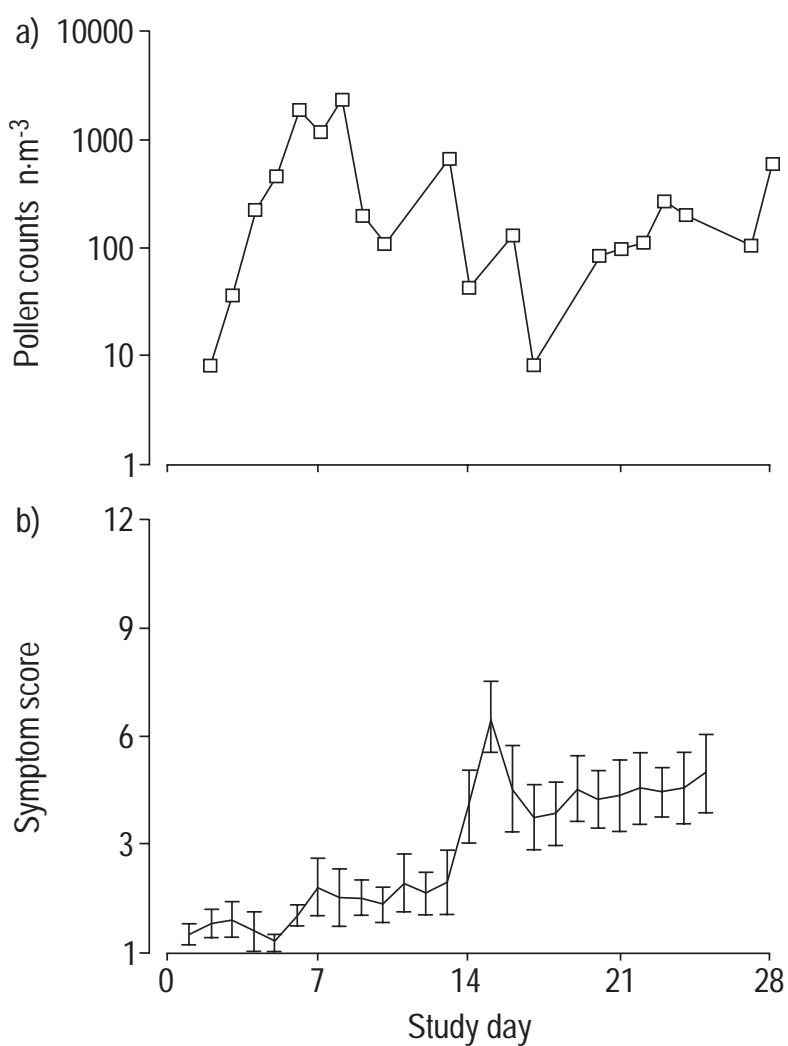

Fig. 1. - a) Pollen counts (registered about $40 \mathrm{~km}$ from the actual study area) and b) nasal symptom scores (mean \pm SEM) during the 4-week study period. The counts demonstrated a marked birch pollen season and the scores indicated moderate symptoms. The nasal symptom scores were significantly increased on study days 8,10 and 11 and from the study day 13 throughout the remaining study period $(\mathrm{p}<0.01-0.05$ versus study day 1).

moderate symptoms during the study period (fig. 1b): nasal symptom scores were significantly increased on study days 8, 10 and 11, and from study day 13 throughout the remaining study period $(\mathrm{p}<0.01-0.05$ compared with study day 1). Six of the 10 patients used loratadine infrequently during the pollen season and the mean consumption of loratadine was five tablets during the study period. In one case, loratadine was taken 2 days before the histamine challenges that were carried out during the season, whereas in the other cases $>7$ days elapsed between loratadine intake and histamine challenge. The isotonic saline lavages were performed before and during the pollen season. The histamine challenges were performed before and after about 3 weeks of pollen exposure.

The baseline lavage fluid levels of $\alpha_{2}$-macroglobulin $(\mathrm{p}<0.01-0.05)$ and ECP $(\mathrm{p}<0.05)$ were increased during the pollen season (fig. $2 \mathrm{a}, \mathrm{b}$ ). There was a significant correlation between the lavage fluid levels of these indices $(\mathrm{r}=0.81, \mathrm{p}<0.0001)$ (fig. 3 ).

Histamine produced concentration-dependent increases in nasal lavage fluid levels of $\alpha_{2}$-macroglobulin before and late during the pollen season (table 1). The microvascular exudative responsiveness to histamine before and during the pollen season did not differ. Histamine (40 $\mu \mathrm{g} \cdot \mathrm{mL}^{-1}$ ) increased nasal lavage fluid levels of ECP during the pollen season $(\mathrm{p}<0.05)$, compared with before
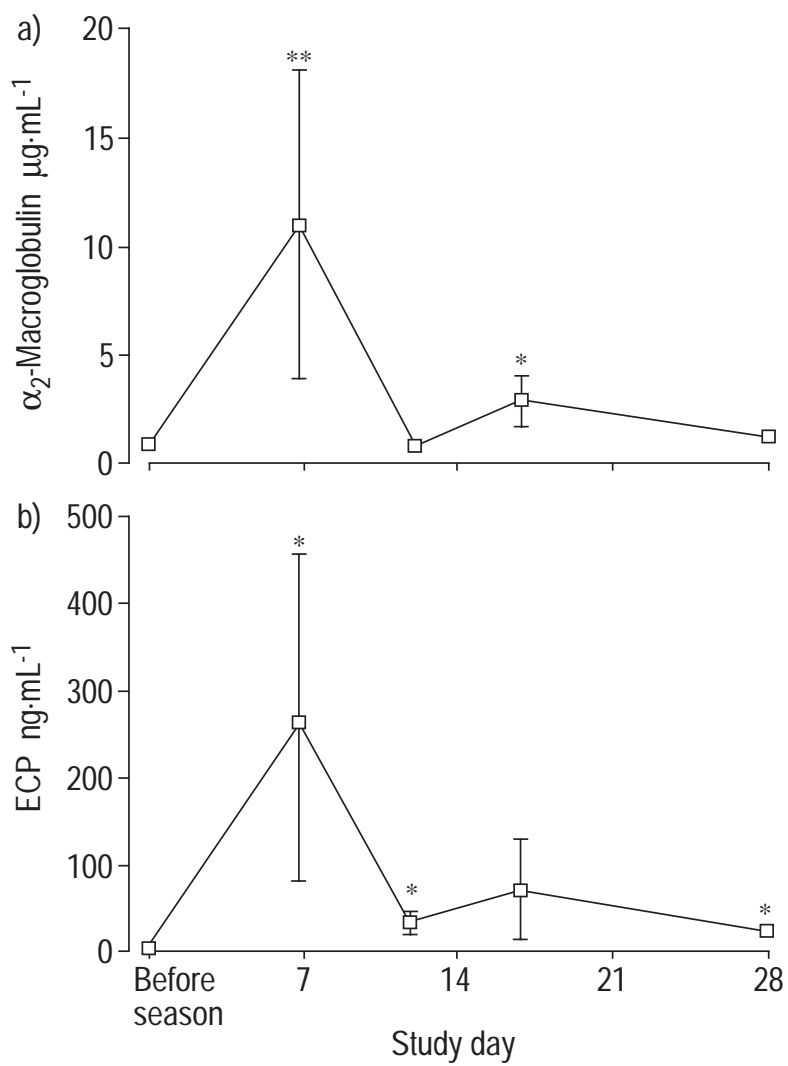

Fig. 2. - Nasal lavage fluid levels (mean \pm SEM) of a) $\alpha_{2}$-macroglobulin and b) eosinophil cationic protein (ECP) recorded before (4-6 weeks before study day 0 ) and on four occasions during the study period. The levels of $\alpha_{2}$-macroglobulin and ECP were significantly elevated during the pollen season $(*: \mathrm{p}<0.05 ; * *: \mathrm{p}<0.01)$.

the season. In contrast, histamine $\left(40 \mu \mathrm{g} \cdot \mathrm{mL}^{-1}\right)$ failed to produce this effect before the season (table 1). Histamine $\left(400 \mu \mathrm{g} \cdot \mathrm{mL}^{-1}\right)$ produced a three-fold, statistically nonsignificant, increase in nasal lavage fluid levels of ECP during the pollen season, compared with before the season, suggesting that the preceding histamine $\left(40 \mu \mathrm{g} \cdot \mathrm{mL}^{-1}\right)$ challenge had partly depleted the mucosa of its free ECP. After histamine challenge, there was a significant correlation between the lavage fluid levels of $\alpha_{2}$-macroglobulin and ECP during the pollen season $(\mathrm{r}=0.40$, $\mathrm{p}<0.05)$ but not before the pollen season.

\section{Discussion}

The present study demonstrated that nasal mucosal exudation of bulk plasma $\left(\alpha_{2}\right.$-macroglobulin) and nasal mucosal output of ECP are significant features in 7-11 yrold children with seasonal allergic rhinitis. The correlation between nasal lavage fluid levels of $\alpha_{2}$-macroglobulin and ECP was excellent and histamine challenge-induced exudation of bulk plasma ( $\alpha_{2}$-macroglobulin) acutely increased the lavage fluid levels of ECP exclusively during the active allergic disease period. These data suggest the participation of activated mucosal eosinophils and exudative processes in childhood seasonal allergic rhinitis and support the notion that exudation of bulk plasma is associated with movement of ECP into the airway lumen during active allergic disease. 


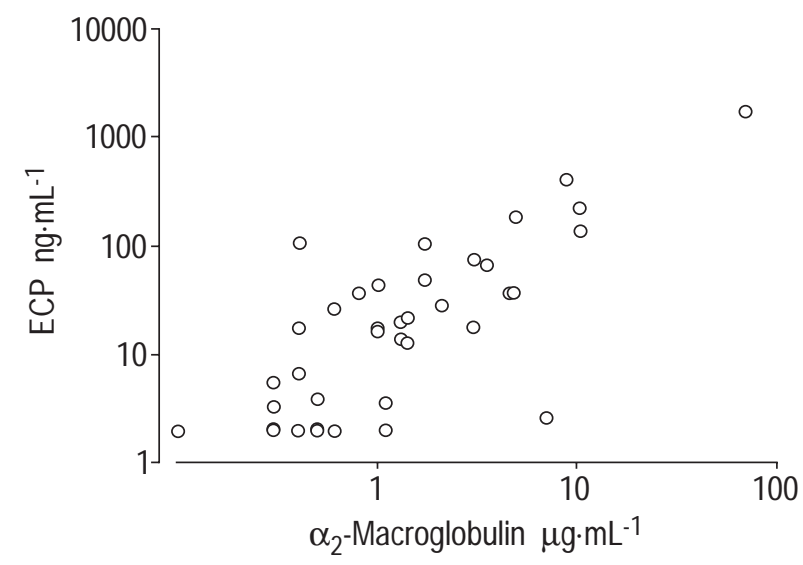

Fig. 3. - Paired nasal lavage fluid levels of $\alpha_{2}$-macroglobulin and eosinophil cationic protein (ECP), demonstrating an excellent correlation between these indices of plasma exudation and eosinophil activation, respectively $(\mathrm{r}=0.81, \mathrm{p}<0.0001)$.

Extravasation, lamina propria flooding and luminal entry of bulk plasma are global features of airway mucosal inflammation in allergic and infectious conditions $[3,5]$. In challenge experiments, it was previously demonstrated that the luminal entry of plasma extends to threshold inflammatory stimulation and that lavage fluid levels of the plasma protein $\alpha_{2}$-macroglobulin $(720 \mathrm{kDa})$ are a particularly valid index of this process $[10,11]$. In the present study, the plasma exudation response to histamine in the allergic mucosa of allergic children was confirmed [4]. Furthermore, the baseline nasal lavage fluid levels of $\alpha_{2-}$ macroglobulin were increased during the pollen season. Mucosal exudation of plasma, determined by minimally invasive methods, may thus be an index of airway inflammation both in children (this study) and in adults $[1,2]$.

Increased nasal surface levels of ECP in children have been demonstrated in atopy [12], respiratory syncytial virus infection [13-16] and allergic rhinitis during seasonal pollen exposure (this study). In the present study, as well as in the previous study in atopic children [12], baseline lavage fluids were frozen without prior centrifugation. Hence, the presence of ECP in these samples could reflect free ECP as well as ECP released from intact eosinophils by the freezing procedure. The present samples obtained after histamine challenge were centrifuged and the presence of eosinophil cells should, therefore, not be an artefact source of ECP in these samples, which have produced the main findings in the present study. However, one cannot exclude the contribution of ECP contained in free eosinophil granules that would remain in samples after centrifugation. Eosinophil cytolysis, generating clusters of free eosinophil granules, emerges as an important in vivo mechanism for eosinophil activation in allergic and nonallergic airway mucosa [17-19] and free eosinophil granules may also occur in nasal secretions produced after nasal allergen exposure [19]. Hence, the present baseline ECP levels may reflect a combination of actual released ECP and released specific granules containing ECP. However, the samples obtained in this study after histamine challenge demonstrated a seasonal increase in ECP which was beyond that observed in the baseline lavage fluids obtained during the season. The ECP that was brought to the surface by an acute histamine challenge (which produced an acute plasma exudation response) would probably reflect already released ECP molecules, since there is no known mechanism by which histamine itself would cause lumenal entry of clusters of free eosinophil granules or cause cellular release of ECP. This reasoning is analogous to the explanation of the finding that interleukin-6, known to be bound to $\alpha_{2}$-macroglobulin [8], can acutely be moved to the airway surface by nasal histamine challenge in adult individuals with allergic rhinitis [5]. As a follow-up to the present study additional data have been obtained suggesting that induced sputum can be combined with induced bronchial exudation to improve significantly the yield and relevance of bronchial mucosal indices of inflammation such as ECP and cytokines [20]. Exudative challenges (such as histamine) in combination with airway lavage and other surface sampling techniques may be important experimental tools to monitor events occurring within the airway mucosal tissues.

In the present study, nasal lavage fluid levels of $\alpha_{2}-$ macroglobulin and ECP were found to be well correlated. Both at baseline (during the season) and after acute histamine challenges (during the season but not before the season) the plasma exudation response was thus associated with the lumenal entry of ECP. These data support the hypothesis that ECP, potentially bound to plasma proteins such as $\alpha_{2}$-macroglobulin, is transported into the airway lumen from the epithelial lining and the lamina propria, where it is released into the extracellular matrix during seasonal pollen exposure. The possibility that eosinophilreleased products increase microvascular permeability to macromolecules [21] may increase the possibility of an association between ECP and $\alpha_{2}$-macroglobulin. Taken together, the present findings support the notion [9] that plasma-derived binding proteins may pick up mucosal ECP molecules and move them to the airway surface. On a

Table 1. - Effects of nasal histamine challenge on nasal lavage fluid levels of $\alpha_{2}$-macroglobulin and eosinophil cationic protein (ECP) before and during the pollen season

Study period

\begin{tabular}{|c|c|c|c|c|}
\hline \multirow{2}{*}{$\begin{array}{l}\text { Histamine } \\
\mu \mathrm{g} \cdot \mathrm{mL}^{-1}\end{array}$} & \multicolumn{2}{|c|}{ Before season } & \multicolumn{2}{|c|}{ During season } \\
\hline & $\underset{\mu \mathrm{g} \cdot \mathrm{mL}^{-1}}{\alpha_{2} \text {-macroglobulin }}$ & $\begin{array}{c}\mathrm{ECP} \\
\mathrm{ng} \cdot \mathrm{mL}^{-1}\end{array}$ & $\underset{\mu \mathrm{g} \cdot \mathrm{mL}^{-1}}{\alpha_{2} \text {-macroglobulin }}$ & $\begin{array}{c}\mathrm{ECP} \\
\mathrm{ng} \cdot \mathrm{mL}^{-1}\end{array}$ \\
\hline 0 & $2.3 \pm 1.6$ & $13.1 \pm 10.8$ & $0.7 \pm 0.3$ & $12.5 \pm 9.6$ \\
\hline 40 & $4.8 \pm 1.9$ & $5.2 \pm 3.1$ & $3.2 \pm 0.7 * *$ & $18.2 \pm 12.9^{\#}$ \\
\hline 400 & $15.4 \pm 4.3 *$ & $3.3 \pm 0.9$ & $8.1 \pm 1.5^{* *}$ & $10.7 \pm 7.9$ \\
\hline
\end{tabular}

Data are shown as mean \pm SEM. ${ }^{*}: \mathrm{p}<0.05$ versus baseline; ${ }^{* *}: \mathrm{p}<0.01$ versus baseline. ${ }^{\#}: \mathrm{p}<0.05$ versus before season. Baseline is histamine $\left(0 \mathrm{mg} \cdot \mathrm{mL}^{-1}\right)$. 
general note, measurements representing tissue ECP may be more relevant to bioactivity than any measurement in the "waste" and degenerating material that may be found in the airway lumen. Hence, the present method may be useful in determining drug effects on eosinophilic nasal inflammation in children.

At present, little is known about the effectiveness of an acute plasma exudation response with regard to "rinsing" the mucosal tissue of molecules such as ECP. The present data suggest that most of the movable ECP required only a low and moderately effective exudative dose of histamine, since a subsequent challenge with a 10 -fold greater concentration of histamine produced a smaller increase in the lumenal ECP. It is inferred from the present data and the hypothesis that ongoing plasma exudation in the airways may be of importance for the appearance of many indices in the lumen and, equally, for the absence of those same indices in tissue samples. For example, it would have been of interest to know the allergen challenge-induced exudative activity in the study by WoOLley et al. [22], who found significant increases in granulocyte-macrophage colony stimulating factor in the bronchial lumen but, curiously, a decrease in the biopsy.

In conclusion, the present data indicate that seasonal allergic rhinitis in 7-11 yr-old children is associated with nasal mucosal exudation of bulk plasma and eosinophil cationic protein, indicating active eosinophilic inflammation in the nasal mucosa of these patients. Furthermore, the data support the hypothesis that the plasma exudation process plays a role in transferring cellular products (notably eosinophil cationic protein) from airway tissue compartments into the airway lumen. Hence, nasal histamine challenges may allow measurements of mucosal tissue molecules such as eosinophil cationic protein in airway surface samples. To some extent the combined exudative challenge and lavage method may thus be a substitute for mucosal biopsies, which is a particularly important consideration in studies involving children. This present study obtained important evidence on nasal eosinophil cationic protein levels by the use of histamine challenge in these young patients.

\footnotetext{
Acknowledgements. The authors thank L. Glans, E. Andersson and B. Holmskov for expert laboratory assistance.
}

\section{References}

1. Naclerio RM, Meier HL, Kagey-Sobotka A, et al. Mediator release after nasal airway challenge with allergen. Am Rev Respir Dis 1983; 128: 597-602.

2. Svensson C, Andersson M, Persson CGA, Venge P, Alkner U, Pipkorn U. Albumin, bradykinins, and eosinophil cationic protein on the nasal mucosal surface in patients with hay fever during natural allergen exposure. $J$ Allergy Clin Immunol 1990; 85: 828-833.

3. Persson CGA, Svensson C, Greiff L, et al. Use of the nose to study the inflammatory response in the respiratory tract. Thorax 1992; 47: 993-1000.

4. Greiff L, Meyer P, Svensson C, Persson CGA, Andersson M. The "Nasal Pool"-device for challenge and lavage of the nasal mucosa in children: histamine-induced plasma exudation responses. Pediatr Allergy Immunol 1997; 8: 137-142.
5. Persson CGA, Erjefält JS, Greiff L, et al. Plasma-derived proteins in airway defence, disease and repair of epithelial injury. Eur Respir J 1998; 11: 958-970.

6. Erjefält JS, Erjefält I, Sundler F, Persson CGA. Epithelial pathways for luminal entry of bulk plasma. Clin Exp Allergy 1995; 25: 197-195.

7. Peterson CG, Venge P. Interaction and complex-formation between the eosinophil cationic protein and $\alpha_{2}$-macroglobulin. Biochem J 1987; 245: 781-787.

8. James K. Interactions between cytokines and $\alpha_{2}$-macroglobulin. Immunol Today 1990; 11: 163-166.

9. Persson CGA, Erjefält JS, Andersson M, et al. Epithelium, microcirculation, and eosinophils - new aspects of the allergic airway in vivo. Allergy 1997; 52: 241-255.

10. Erjefält I, Persson CGA. Inflammatory passage of plasma macromolecules into airway wall and lumen. Pulm Pharmacol 1989; 2: 93-102.

11. Svensson C, Andersson M, Greiff L, Alkner U, Persson CGA. Exudative hyperresponsiveness of the airway microcirculation in seasonal allergic rhinitis. Clin Exp Allergy 1995; 25: 942-950.

12. Noah TL, Henderson FW, Henry MM, Peden DP, Devlin RB. Nasal lavage cytokines in normal, allergic, and asthmatic school-age children. Am J Respir Crit Care Med 1995; 152: 1290-1296.

13. Sigurs N, Bjarnason R, Sigurbergsson F. Eosinophil cationic protein in nasal secretion and in serum and myeloperoxidase in serum in respiratory syncytial virus bronchiolitis: relation to asthma and atopy. Acta Paediatr 1994; 83: 1151-1155.

14. Garofalo R, Dorris A, Ahlstedt S, Welliver RC. Peripheral blood eosinophil counts and eosinophil cationic protein content of respiratory secretions in bronchiolitis: relationship to severity of disease. Pediatr Allergy Immunol 1994; 5: 111-117.

15. Colocho-Zelaya EA, Örvell C, Strannegärd Ö. Eosinophil cationic protein in nasopharyngeal secretions and serum of infants infected with respiratory syncytial virus. Pediatr Allergy Immunol 1994; 5: 100-106.

16. IngramJM, Rakes GP, Hoover GE, Platts-Mills TAE, Heyman PW. Eosinophil cationic protein in serum and nasal washes from wheezing infants and children. $J$ Pediatr 1995; 127: 558-564.

17. Persson CGA, Erjefält JS. Eosinophil lysis and free granules: an in vivo paradigm for cell activation and drug development. Trends Pharmacol Sci 1997; 18: 117-123.

18. Greiff L, Erjefält JS, Andersson M, Svensson C, Persson CGA. Generation of clusters of free eosinophil granules (Cfegs) in seasonal allergic rhinitis. Allergy 1998; 53: 200-203.

19. Erjefält JS, Greiff L, Korsgren M, Persson G, Sundler F, Persson CGA. Eosinophil lysis and clusters of free eosinophil granules (Cfegs) in rhinitis and asthma. $\mathrm{Am} \mathrm{J}$ Respir Crit Care Med 1997; 155: A977.

20. Greiff L, Andersson M, Halldorsdottir H, et al. Bronchial hyperreactivity (BHR) and sputum indices of inflammation in seasonal allergic rhinitis (SAR). Am J Respir Crit Care Med 1997; 155: A980.

21. Minnicozzi M, Duran WN, Gleich GJ, Egan RW. Eosinophil granule proteins increase microvascular macromolecular transport in the hamster cheek pouch. $J$ Immunol 1994; 153: 2664-2670.

22. Woolley KL, Ädelroth E, Woolley MJ, Ellis R, Jordana M, O'Byrne PM. Effect of allergen challenge on eosinophils, eosinophil cationic protein, granulocyte-macrophage colony-stimulating factor in mild asthma. $A m J$ Respir Crit Care Med 1995; 151: 1915-1924. 